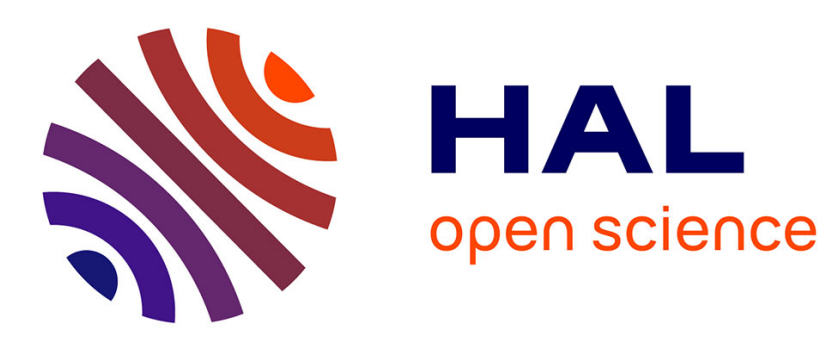

\title{
Pore network modelling of condensation in gas diffusion layers of proton exchange membrane fuel cells
}

\author{
Benjamin Straubhaar, Joël Pauchet, Marc Prat
}

\section{To cite this version:}

Benjamin Straubhaar, Joël Pauchet, Marc Prat. Pore network modelling of condensation in gas diffusion layers of proton exchange membrane fuel cells. International Journal of Heat and Mass Transfer, 2016, vol. 102, pp. 891-901. 10.1016/j.ijheatmasstransfer.2016.06.078 . hal-01373944

\section{HAL Id: hal-01373944 \\ https://hal.science/hal-01373944}

Submitted on 29 Sep 2016

HAL is a multi-disciplinary open access archive for the deposit and dissemination of scientific research documents, whether they are published or not. The documents may come from teaching and research institutions in France or abroad, or from public or private research centers.
L'archive ouverte pluridisciplinaire $\mathbf{H A L}$, est destinée au dépôt et à la diffusion de documents scientifiques de niveau recherche, publiés ou non, émanant des établissements d'enseignement et de recherche français ou étrangers, des laboratoires publics ou privés. 


\section{Open Archive TOULOUSE Archive Ouverte (OATAO)}

OATAO is an open access repository that collects the work of Toulouse researchers and makes it freely available over the web where possible.

This is an author-deposited version published in : http://oatao.univ-toulouse.fr/ Eprints ID : 16181

To link to this article : DOI:10.1016/j.ijheatmasstransfer.2016.06.078 URL : http://dx.doi.org/10.1016/j.ijheatmasstransfer.2016.06.078

To cite this version : Straubhaar, Benjamin and Prat, Marc and Pauchet, Joël Pore network modelling of condensation in gas diffusion layers of proton exchange membrane fuel cells. (2016) International Journal of Heat and Mass Transfer, vol. 102. pp. 891-901. ISSN 00179310

Any correspondence concerning this service should be sent to the repository administrator: staff-oatao@listes-diff.inp-toulouse.fr 


\title{
Pore network modelling of condensation in gas diffusion layers of proton exchange membrane fuel cells
}

\author{
B. Straubhaar ${ }^{\mathrm{a}, \mathrm{b}}$, J. Pauchet ${ }^{\mathrm{c}}$, M. Prat ${ }^{\mathrm{a}, \mathrm{b}, *}$ \\ a INPT, UPS, IMFT (Institut de Mécanique des Fluides de Toulouse), Université de Toulouse, Allée Camille Soula, F-31400 Toulouse, France \\ ${ }^{\mathrm{b}}$ CNRS, IMFT, F-31400 Toulouse, France \\ ' CEA, LITEN, LCPEM, Laboratory of Fuel Cell Components, Electrolysers and Modeling, 17 rue des Martyrs, 38054 Grenoble, France
}

Keywords:

Pore network modelling

Condensation

Evaporation

Temperature gradient

Gas diffusion layer

\begin{abstract}
A B S T R A C T
A pore network model (PNM) is exploited to simulate the liquid water formation by vapour condensation in the gas diffusion layer (GDL) on the cathode side considering the spatial temperature variations within the GDL. The computed distributions are markedly different from the ones computed in previous works assuming capillarity controlled invasion in liquid phase from the catalyst layer and found to be in quite good agreement with several experimental observations. The proposed model opens up new perspectives for understanding the water transfer in protons exchange membrane fuel cells and the associated water management and aging issues.
\end{abstract}

\section{Introduction}

In spite of many studies, the exact mechanisms of water transfer in the various layers forming a proton exchange membrane fuel cell (PEMFC) are not fully understood. This also holds for the gas diffusion layers (GDL).

As discussed in [1], different mechanisms can be invoked as regards the transfer across the GDL of the water generated by the electrochemical reaction in the catalyst layer $(\mathrm{CL})$ : transfer in vapour phase, transfer in liquid phase with negligible liquidvapour phase change phenomena, transfer with evaporation condensation mechanisms. This is so because we actually do not know whether the water formed in the CL enters the GDL in liquid phase, in vapour phase or as a mix of both phases. This fundamental question is schematically illustrated in Fig. 1. Although detailed in-situ visualizations of liquid phase distributions in a GDL are possible using for example mini PEMFC dedicated for X-ray tomography, e.g. [2], it is difficult to infer from the visualizations the exact mechanisms at play. We can therefore distinguish two assumptions. Assumption \#1 consists in assuming that water enters the GDL in liquid phase. Assumption \#2 consists in assuming that water enters the GDL in vapour phase. Since it is difficult to

\footnotetext{
* Corresponding author at: INPT, UPS, IMFT (Institut de Mécanique des Fluides de Toulouse), Université de Toulouse, Allée Camille Soula, F-31400 Toulouse, France. E-mail address: mprat@imft.fr (M. Prat).
}

decide from the available experimental data which assumption is the most relevant, additional useful insights can be sought from modelling and numerical simulations.

As for other problems involving multiphase flows in porous media, many techniques have been used in relation with PEM fuel cells. The most frequent one is based on the classical continuum approach to porous media and involves the generalized Darcy's law and the concepts of capillary pressure curves and relative permeabilities. Although widely used, notably in CFD commercial codes, the relevance of this classical approach has been questioned, e.g. [3], because this type is modelling is not well adapted to simulate the capillary forces dominated regime prevailing in GDL. There is also a problematical lack of length scale separation since the thickness of a GDL is typically less than 10 pore sizes. Owing to these limitations, alternative approaches have been developed. These notably include the direct simulations, such the ones based on Lattice Boltzmann Methods, e.g. [4], Monte Carlo simulations [5] and the simulations based on pore network models (PNM). The computational times of the latter over domains of comparable sizes, i.e. containing the same number of pores, are typically orders of magnitude smaller than for the two other methods. For this reason as well as the general simplicity of this approach, the present study is based on a PNM approach.

PNMs have become a somewhat popular tool to study transfer phenomena in GDL, e.g. [6-23]. It must be noted that these studies are all more or less explicitly based on assumption \#1, i.e. all the 


\begin{tabular}{|c|c|c|c|}
\hline \multicolumn{4}{|c|}{ Nomenclature } \\
\hline$a$ & lattice spacing, m & $U$ & electrical tension, $\mathrm{V}$ \\
\hline$c$ & gas phase mole concentration, $\mathrm{mol} / \mathrm{m}^{3}$ & $V_{p}$ & pore volume, $\mathrm{m}^{3}$ \\
\hline$d$ & throat width, m & $x_{v}$ & vapour mole fraction \\
\hline$D$ & vapour diffusion coeff., $\mathrm{m}^{2} / \mathrm{s}$ & & \\
\hline$D^{*}$ & vapour diffusion effective coefficient, $\mathrm{m}^{2} / \mathrm{s}$ & \multicolumn{2}{|c|}{ Greek symbols } \\
\hline$F$ & Faraday constant, C & $\beta$ & partition coefficient \\
\hline$g$ & throat diffusive conductance, $\mathrm{mol} / \mathrm{s}$ & $\delta$ & GDL thickness, $\mathrm{m}$ \\
\hline$H_{c h}$ & channel height, m & $\varepsilon$ & porosity \\
\hline$h_{l v}$ & reaction enthalpy, $\mathrm{J} / \mathrm{mol}$ & $\Phi$ & heat flux, $\mathrm{W} / \mathrm{m}^{2}$ \\
\hline$i$ & current density, $\mathrm{A} / \mathrm{cm}^{2}$ & $\lambda$ & thermal conductivity, $\mathrm{W} / \mathrm{m} / \mathrm{K}$ \\
\hline$L$ & GDL lateral size, $\mathrm{m}$ & $\lambda^{*}$ & effective thermal conductivity, $\mathrm{W} / \mathrm{m} / \mathrm{K}$ \\
\hline$L_{c}$ & channel width, m & $\eta$ & nucleation parameter \\
\hline$L_{r}$ & rib width, m & $\chi$ & pore volume correction factor \\
\hline $\mathbf{n}$ & unit normal vector & & \\
\hline & vapour partial pressure, $\mathrm{Pa}$ & \multicolumn{2}{|c|}{ Subscripts } \\
\hline & saturation vapour pressure, $\mathrm{Pa}$ & $b p$ & bipolar plate \\
\hline$P_{\text {ref }}$ & gas phase total pressure, $\mathrm{Pa}$ & $c$ & compressed \\
\hline$Q$ & water injection flux, $\mathrm{mol} / \mathrm{m}^{2} / \mathrm{s}$ & ch & channel \\
\hline$R$ & gas constant, $\mathrm{J} / \mathrm{kg} / \mathrm{K}$ & $p$ & pore \\
\hline RH & local relative humidity, \% & $u c$ & uncompressed \\
\hline$R H_{c h}$ & channel relative humidity, \% & $v$ & vapor \\
\hline$T$ & temperature, $\mathrm{K}$ & 11 & in plane \\
\hline$T_{b p}$ & bipolar plate temperature, $\mathrm{K}$ & $\begin{array}{l}11 \\
\perp\end{array}$ & through plane \\
\hline
\end{tabular}

water coming from the adjacent catalyst layer enters the GDL in liquid phase and flows through the GDL in liquid phase. Assumption \#1 of liquid water invasion is also made in [24], but with the consideration of an additional phenomenon, the possible evaporation of the liquid within the GDL, see also [1]. In brief, to the best of our knowledge, no previous study based on PNM has explicitly considered assumption \#2 as central assumption. In contrast with the aforementioned studies, the present work is based on assumption \#2, i.e. we explore the situation where water enters the GDL in vapour phase from the CL.

As we shall see, the occurrence of water in the GDL under assumption \#2 is then due to the condensation of the water vapour.
Modelling the condensation process in a GDL using PNM or related approaches is not completely a novelty. For instance, one can cite the qualitative studies reported in $[25,26]$ limited to 2D simulations and without explicit consideration of the temperature variations across the GDL, a key aspect, however, for the simulation of condensation, and our recent paper [1], where a condensation algorithm was presented and briefly illustrated through a few simulations in a 2D pore network only. The fact that previous PNM studies dealing with condensation are scarce and somewhat skimpy is somewhat surprising since condensation is considered as an essential process by several authors, e.g. [26-34].

The objective of the present article is therefore to present and analyse PNM simulations of liquid water formation by

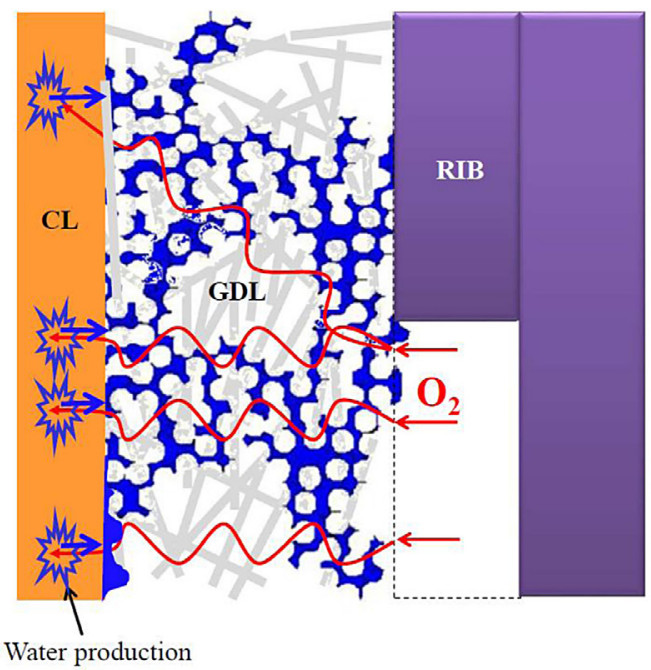

(a)

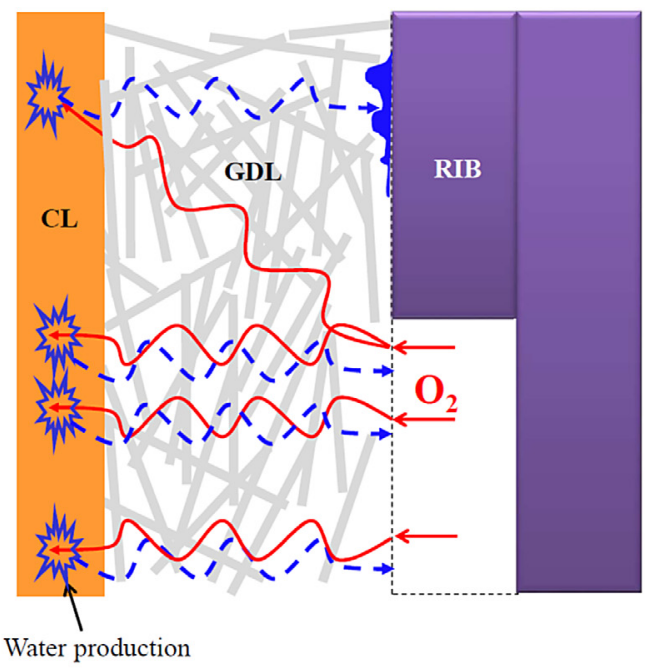

(b)

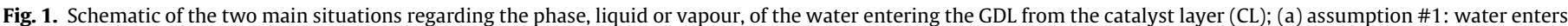

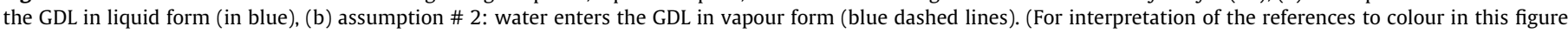
legend, the reader is referred to the web version of this article.) 
condensation in a GDL under assumption \#2. It should be pointed out that the consideration of the condensation process is intimately related to the consideration of a non-uniform temperature field across the GDL. This is in contrast with most of previous studies of GDL based on PNM, where the temperature was in fact more or less implicitly assumed uniform and constant over the GDL. Actually, there are clear indications that the average temperature is greater at the GDL - catalyst layer interface than at the GDL bipolar plate interface and greater at the channel - GDL interface than right below the ribs of bipolar plate, e.g. [28], [32-34]. The existence of a colder region below the ribs clearly suggests a possibility of condensation.

In this context, an important aspect will be to explore how the PNM condensation simulations compared with the in-situ behaviours and data obtained experimentally as reported for instance in [35-37] with the tentative objective of elucidating the mechanisms leading to the occurrence of liquid water in GDL.

The paper is organized as follows. The computational domain and boundary conditions for the mass transfer problem are described in Section 2. The heat transfer problem is described in Section 3. The pore network is presented in Section 4. The pore network condensation algorithm is presented in Section 5. The condensation diagram is presented in Section 6. Liquid water distribution is discussed in Section 7 from a series of PNM simulations. A short discussion is open in Section 8. A conclusion is presented in Section 9.

\section{GDL unit cell and water transport boundary conditions}

\subsection{Polarization curve. GDL unit cell}

A PEMFC is globally characterized by its polarization curve. For the present study we adopt the polarization curve depicted in Fig. 2. It corresponds to the polarization curve presented in [35].

The GDL properties are specified from data obtained within the framework of IMPALA European project (see acknowledgement section) or from the literature. They correspond to SGL 24BA. These properties are specified as best as we can from available data and then the simulations are run. In other terms, no parameter adjustment is performed. These properties are given below (geometrical properties) as well as in Sections 3 (throat size distributions), 4 (thermal properties) and 5 (diffusion properties).

The GDL is supposed to be hydrophobic, i.e. the fibrous matrix is supposed carpeted by PTFE (the contact angle of liquid water on

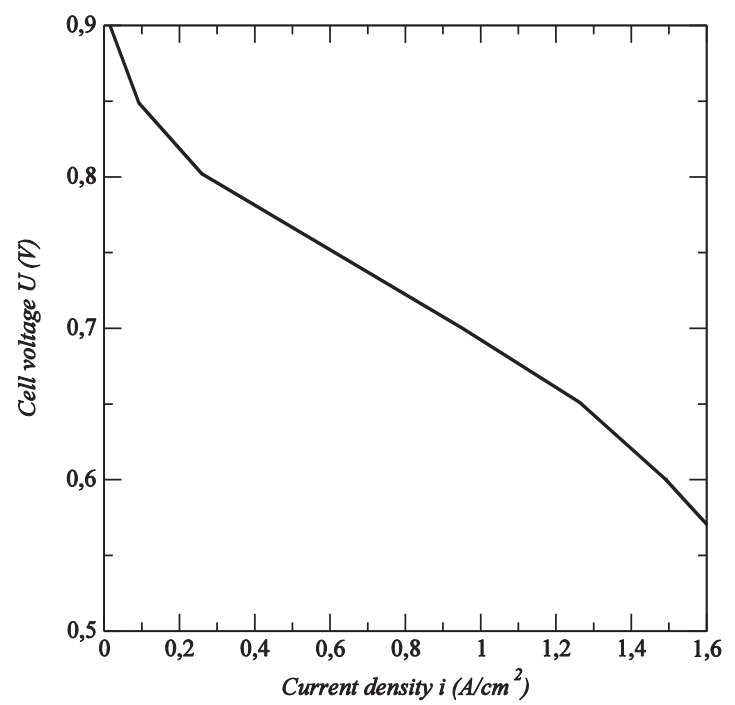

Fig. 2. The polarization curve used throughout this paper (adapted from [35]).

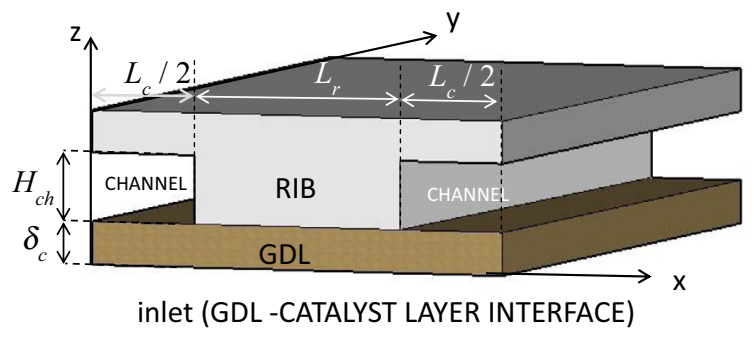

Fig. 3. GDL unit cell with one rib and two half channels.

PTFE is about $110^{\circ}$ ). As in many previous studies, only a sub-region of the GDL on the cathode side is considered. This sub-region is illustrated in Fig. 3 and referred to as a GDL unit cell. As sketched in Fig. 3, the in-plane transverse size $L$ of the unit cell in the x-direction is equal to the cumulated widths $L_{r}+L_{c}$ of one rib and one channel. We take representative values, namely $L_{r}=0.96 \mathrm{~mm}$ and $L_{c}=0.96 \mathrm{~mm}$ leading to $L=1.92 \mathrm{~mm}$. The in plane size $L_{y}$ of the considered GDL sub-region in the $y$ direction is $L_{y}=2.96 \mathrm{~mm}$. The latter is actually unnecessarily large. Taking $L_{y}=L$ would have been sufficient and would have led to the same results. The GDL is thin. We take for its thickness $\delta_{c}=120 \mu \mathrm{m}$. This corresponds to the GDL below the rib, which is the compressed GDL (as indicated by the subscript " $\mathrm{c}$ "). The uncompressed GDL thickness is $\delta_{u c}=190 \mu \mathrm{m}$ (using the subscript "uc" for properties of the uncompressed region, i.e. below the channel). Different GDL properties are specified in the region below the rib and in the regions below the channels so as to take into account the differential compression of the GDL. For example, the porosity of the uncompressed GDL (regions below the channels) is $\varepsilon_{u c}=74 \%$. The porosity of the compressed GDL (region below the rib) is estimated from the relationship $\varepsilon_{c}=1-\frac{\delta_{u c}}{\delta_{c}}\left(1-\varepsilon_{u c}\right)$. This gives $\varepsilon_{C}=59 \%$.

The channel height is $H_{c h}=0.48 \mathrm{~mm}$. As can be seen, the rib is at the centre of the GDL outlet. The GDL unit cell inlet corresponds to the GDL-catalyst layer interface (or the micro porous layer (MPL) - GDL interface if a MPL is assumed to be present).

\subsection{Boundary conditions for the water transport}

The relative humidity and gas total pressure at the outlet of unit cell $\left(z=\delta_{c}\right)$ are input parameters. They are denoted by $R H_{c h}$ and $P_{r e f}$ respectively. We take $P_{\text {ref }}=1.5$ bar. $R H_{c h}$ is varied. At the GDL inlet $(z=0)$, a water injection flux $\left(\mathrm{mol} \cdot \mathrm{m}^{-2} \cdot \mathrm{s}^{-1}\right)$ is imposed.

It is classically expressed as a function of local current density $i$ as

$Q=\beta \frac{i}{2 F}$

where $F$ is the Faraday's constant ( $F=96485.34 \mathrm{C})$.

Note again that it is assumed that water is injected in vapour form and not in liquid form. Actually not all the water produced at the cathode is transported toward the GDL. A fraction is transported in direction of the membrane. The coefficient $\beta$ in Eq. (1) is the fraction directed toward the cathode GDL. Throughout this paper, we make the simplifying assumption that this fraction is independent of $i$ and take $\beta=0.8$.

The boundary conditions for the water transport are summarized in Fig. 4 together with the heat transfer problem.

\section{Heat transfer}

The temperature field $T(x, y, z)$ within the GDL is a crucial information for the computation of liquid water formation by 


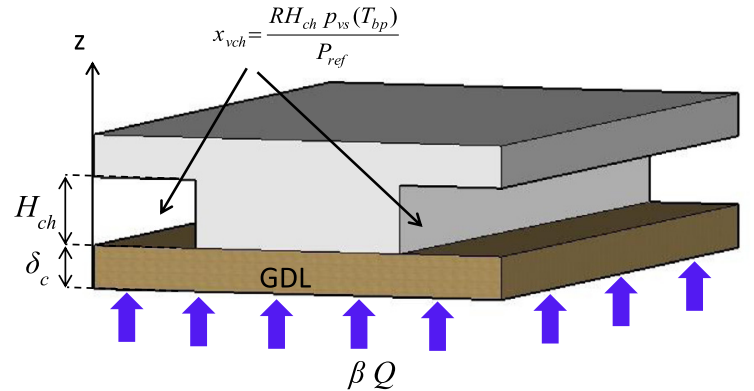

(a)

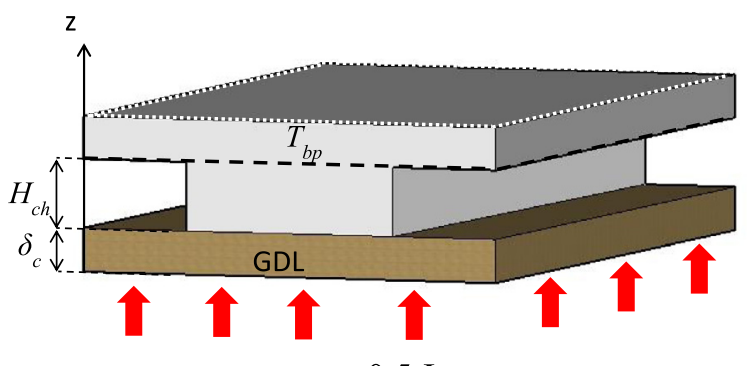

$0.5 \Phi$

(b)

Fig. 4. Schematic drawing of studied problem. (a) water transfer problem: a fraction of the water produced in the catalyst layer is directed toward the cathode GDL; given relative humidity and total gas pressure are imposed in the channels, (b) heat transfer problem: a heat flux is applied at the GDL inlet whereas the temperature $T_{b p}$ is imposed all over the surface in the $x-y$ plane at the position $z=\delta_{c}+H_{c h}$.

condensation. This field is determined numerically by solving the steady state heat conduction problem,

$\nabla \cdot(\lambda \cdot \nabla T)=0$.

in the domain depicted in Fig. 4(b) using a classical finite volume technique. Note that this problem is actually solved in twodimensions over a cross-section in a $x-z$ plane of the domain shown in Fig. 4(b). Then the temperatures so computed is distributed over the pores of the pore network model (see Section 4).

No flux boundary conditions are imposed on the lateral sides of the domain whereas a uniform temperature $T=T_{b p}$ is imposed along the top boundary in Fig. 4(b). Numerical simulations indicated that it is not necessary to take into account explicitly the part of the bipolar plate above the rib in Fig. 4(b). Owing to the high thermal conductivity of the bipolar plate, it is sufficient to impose the temperature $T_{b p}$ directly at the $x-y$ plane located at the position $z=\delta_{c}+H_{c h}$ in Fig. 4(b).

The electrochemical reaction is exothermic. The corresponding heat production per unit surface area $\left(\mathrm{Wm}^{-2}\right)$ can be expressed as, e.g. [34],

$\Phi=\left(\frac{h_{l v}}{2 F}-U\right) i$

where $h_{l v}$ is the reaction enthalpy assuming that water forms in vapour phase $\left(h_{l v}=242,000 \mathrm{~J} \cdot \mathrm{mol}^{-1}\right), U$ the electrical tension. It can be reasonably assumed that half of the produced heat goes toward the anode and half toward the cathode GDL. Therefore, the boundary condition imposed at the GDL inlet for the heat transfer problem is

$0.5 \Phi=0.5\left(\frac{h_{l v}}{2 F}-U\right) i=-\lambda_{\perp}^{*} \frac{\partial T}{\partial z} \quad$ at $z=0$. where $z$ is a Cartesian coordinate in the through-plane direction ( $z=0$ at the GDL inlet); $\lambda_{\perp}^{*}$ is the GDL effective thermal conductivity in the through plane direction. The GDL is a highly anisotropic material owing to the preferential orientation of the fibres in the in-plane direction with a much higher thermal conductivity in the plane direction. Also, owing to the differential compression of the GDL between the rib and channel, the thermal conductivity is higher under the rib region than under the channel. As representative values of a dry GDL, e.g. [38], we take $\lambda_{\perp u c}^{*}=0.25 \mathrm{Wm}^{-1} \mathrm{~K}^{-1}$ (through-plane direction) and $\lambda_{/ / u c}^{*}=4 \mathrm{Wm}^{-1} \mathrm{~K}^{-1}$ (in-plane direction) in the regions of the GDL under the channel and $\lambda_{\perp c}^{*}=0.25 \mathrm{Wm}^{-1} \mathrm{~K}^{-1}$ (through plane) and $\lambda_{/ / u c}^{*}=6.64 \mathrm{Wm}^{-1} \mathrm{~K}^{-1}$ in the GDL under the rib. The thermal conductivity in the channel is $\lambda_{c h}=0.027 \mathrm{Wm}^{-1} \mathrm{~K}^{-1}$ (air) whereas the thermal conductivity of the bipolar plate is $\lambda_{b p}=150 \mathrm{Wm}^{-1} \mathrm{~K}^{-1}$.

An example of temperature field is shown in Fig. 5. This field is actually obtained with the commercial code Comsol Multiphysics ${ }^{\circledR}$ used to validate our finite volume computation. As can be seen, the temperature field is characterized not only by through plane variations but also by significant in - plane variations. Globally, the region in the GDL underneath the rib is colder than the region under the channel. The hottest region in the GDL is at the GDL $\mathrm{CL}$ interface below the channel whereas the colder spot is in the middle of the rib along the GDL - rib interface. The temperature field shown in Fig. 5 is, however, for a spatially uniform isotropic thermal conductivity $\left(\lambda_{\perp u c}^{*}=\lambda_{/ / u c}^{*}=\lambda_{\perp c}^{*}=\lambda_{/ / c}^{*}=\lambda^{*}=1 \mathrm{Wm}^{-1} \mathrm{~K}^{-1}\right)$. When the anisotropy of the thermal conductivity is taken into account the in-plane temperature variations are significantly less than depicted in Fig. 5.

The order of magnitude of temperature variations depicted in Fig. 5 is consistent with the values reported in the literature, e.g. [28], [34]. All other things being equal, the lower is the thermal conductivity of the GDL, the greater is the temperature variation across the GDL.

For a given current density $i$ and the values of parameters specified in this section, the temperature field within the GDL is computed. This field is considered as input data for the pore network simulation of condensation described in Section 4. Thus it should be clear that the temperature field is computed in a preprocessing step prior to the PNM simulation of condensation.

\section{Pore network structure}

Most of previous works on GDL using PNM have been based on structured pore networks, typically cubic networks such as the one depicted in Fig. 6. An exception is the work presented in [10] where unstructured pore networks were generated using a tessellation technique. It is also possible to construct an unstructured network from X-ray tomography images of GDL microstructure as shown in

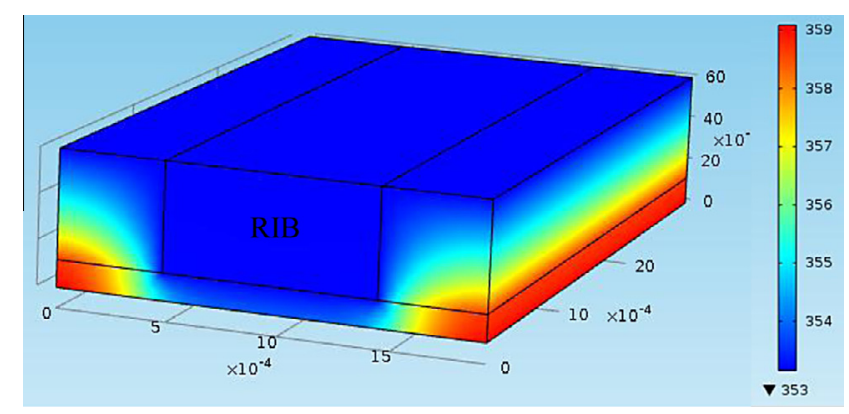

Fig. 5. Example of temperature field (K) computed for $T_{b p}=80^{\circ} \mathrm{C}$ and $i=1 \mathrm{~A} / \mathrm{cm}^{2}$ (which corresponds to0.5 $\left.\Phi=2900 \mathrm{~W} / \mathrm{m}^{2}\right)$ ). 


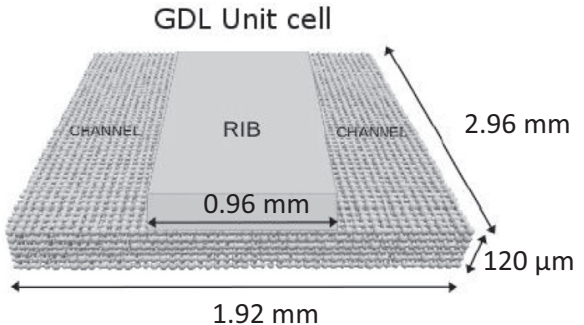

Fig. 6. Sketch of cubic network used to represent the GDL. The lattice spacing $a_{/ /}$ (distance between two neighbor pore) is $80 \mu \mathrm{m}$ in the plane directions. The GDL is represented by a $25 \times 38 \times 4$ cubic network.

[39]. The simple cubic network sketched in Fig. 6 is, however, used for the present work for simplicity. Nevertheless, it should be clear that the condensation algorithm described in Section 4 can be implemented on unstructured networks as well. The GDL pore space is thus represented by a three-dimensional cubic network of pores interconnected with channels, also referred to as throats. The pores are cubic bodies corresponding to the nodes of the network whereas the throats are very short ducts of square crosssection representing the narrower passage between two adjacent pores. The analysis of images of SGL 24BA reported in [40] indicates that the lattice spacing is different in the in-plane direction with approximately $a_{/ /}=80 \mu \mathrm{m}$ from the lattice spacing in the through plane direction with approximately $a_{\perp}=40 \mu \mathrm{m}$. We consider a cubic network with $a=80 \mu \mathrm{m}$ in both the in-plane and through plane directions but we take into account the fact that the real lattice spacing is actually twice as small in the through plane direction by modifying the transport properties adequately (for example the through plane thermal conductivity is multiplied by a factor 2 so as to make the through plane network thermal conductance $\lambda_{\perp} / \delta$ identical to the GDL one). Based on the size of the domain occupied by the GDL in the experiment, this leads to represent the GDL by a $25 \times 38 \times 4$ pore network (the figures indicate the number of pores along the directions of the Cartesian grid shown in Fig. 3). Hence, the GDL unit cell has 4 pores in the through-plane direction.

The throat and pore sizes are specified as follows: Because of the GDL anisotropic fibrous structure throats are narrower by about a factor 2 in the in-plane directions compared to the through plane direction [40]. To reflect this anisotropy, the through plane throat sizes are randomly distributed according to a uniform distribution of mean $\bar{d}_{t \perp c}=43 \mu \mathrm{m}$, in the range $\left[d_{t \perp \min , c}-d_{t \perp \text { max }, c}\right]$ with $d_{t \perp \min , c}=32 \mu \mathrm{m} d_{t \perp \max , c}=54 \mu \mathrm{m}$ whereas the in plane throat sizes are randomly distributed according to a uniform distribution of mean $\bar{d}_{t / / c}=21.5 \mu \mathrm{m}$ in the range $\left[d_{t / / \min , c}-d_{t / / \max , c}\right]$ with $d_{t / / \min , c}=16 \mu \mathrm{m}$ and $d_{t / / \max , c}=27 \mu \mathrm{m}$. It is further assumed that these data corresponds to the compressed GDL (regions under the ribs). The GDL is supposed not compressed below the channel considering that this can be taken into account by assuming that the throat size in the through plane direction are not significantly modified whereas the throat sizes in the in-plane directions are larger on average in the uncompressed regions by a factor $\sqrt{\delta_{u c} / \delta_{c}}=1.14$.

The throats are volumeless. Thus all the pore volume is in the nodes. The throat sizes are first distributed as indicated above depending on the location of the throat (compressed or uncompressed regions) and orientation of the throat (through plane or in plane).The volume of a pore is first simply computed from adjacent throat sizes as $V_{p}=\left(\max \left(d_{t}\right)\right)^{3}$ where $\max \left(d_{t}\right)$ is the size of the largest throat to which the pore is connected. Then the pore volumes are corrected, i.e. multiplied by a correction factor, so as to obtain the desired porosity in the considered region $\left(\varepsilon_{u c}=74 \%\right.$ in the uncompressed regions, $\varepsilon_{C}=59 \%$ in the compressed regions). The correction is as follows.The total pore volume is $V_{\text {pcomp }}=\sum_{i=1}^{n_{p c}} V_{p i}$ in the compressed region (where there are $n_{p c}$ pores). The real pore volume is $V_{\text {pcomp,exp }}=\varepsilon_{c} V_{\text {comp }}$ where $V_{\text {comp }}$ is the volume of the region defined as the compressed GDL. $V_{\text {comp }}=$ rib surface area $\times \delta_{c}$. This allows introducing a correction factor defined as $\chi=\frac{V_{\text {pcomp, exp }}}{V_{\text {pcomp }}}$, which is applied to each pore of the considered compressed region, $V_{\text {pinew }}=\chi V_{\text {pi }}$.In this way, the porosity in the network has the desired value. A similar procedure is applied to the uncompressed regions (where $\varepsilon=\varepsilon_{u c}=74 \%$ ).

\section{Pore network model of condensation (and evaporation)}

Two main steps must be distinguished to simulate the vapour condensation process in the network: the nucleation step and the growth step.

\subsection{Nucleation step}

The objective of the nucleation step is to determine points in the network where the condensation is likely to start. The procedure begins by determining the vapour partial pressure field in the network assuming no condensation. To this end, we solved the diffusion problem governing the vapour transport within the network. The transport of water vapour in the GDL is modelled using Fick's law. Expressed in terms of computation on a pore network, the vapour diffusion flux between two nodes (pores) $i$ and $j$ of network is therefore expressed as

$N_{A}=g_{i j}\left(x_{v, j}-x_{v, i}\right)$

where $x_{v}$ is the mole fraction of water vapour and $g_{i j}$ is the diffusive conductivity of the throat connecting the two pores. Traditionally, e.g. [7], $g_{i j}$ is expressed as a function of the throat cross section area (i.e. $d_{t, i j}^{2}$ for a throat of square cross-section), which means that $g_{i j}$ varies (randomly) from one throat to the other. Here we proceed differently following the concepts of the so-called mixed pore network presented in [41]. The local conductance does not vary randomly anymore and is expressed as indicated in Table 1. In Table $1, c$ is the mole concentration of the gas phase: $c \approx \frac{P_{\text {ref }}}{R T_{b p}}$ where $R$ is the ideal gas constant. Note that the total pressure is supposed uniform and constant over the computational domain and equal to $P_{\text {ref. }}$

Table 1 Expression of throat diffusive conductivity in the various regions of GDL; $a_{/ /}=80 \mu \mathrm{m} ; a_{\perp}=40 \mu \mathrm{m}$.

\begin{tabular}{lll}
\hline Region & direction & \\
\hline Compressed & Through-plane & $g_{\perp c}=c \frac{a_{\| /}^{2}}{a_{\perp}} D_{\perp c}^{*}$ \\
Compressed & In-plane & $g_{/ / c}=c a_{\perp} D_{/ / c}^{*}$ \\
Uncompressed & Through-plane & $g_{\perp \text { unc }}=c a_{/ /}^{2} \frac{D_{\perp u c}^{*} a_{\perp}}{a_{u c}}$ \\
Uncompressed & In-plane & $g_{/ / \text {unc }}=c a_{\perp} \frac{\delta_{u c}}{\delta_{c}} D_{/ / u c}^{*}$ \\
\hline
\end{tabular}

Coefficients $D_{\perp c}^{*}, D_{/ / c}^{*}, D_{\perp u c}^{*}, D_{/ / u c}^{*}$ in Table 1 are the GDL effective diffusion coefficients in the through-plane direction (subscript “ $\perp$ ”) and in the in-plane direction (subscript "//") for the GDL compressed region and uncompressed region. Hence, an advantage of the mixed pore network formulation is to directly express the local conductance as a function of the medium effective transport properties. We take $D_{\perp c}^{*} / D=0.25$, where $D$ is the binary diffusion coefficient. In spite of narrower passages in the in-plane directions, the effective diffusion coefficient in this direction is greater than the effective diffusion coefficient in the through plane 
Table 1

Parameters of the reference simulation.

\begin{tabular}{|c|c|c|c|c|c|c|c|c|c|c|c|}
\hline$\lambda_{\perp u c}^{*} \mathrm{Wm}^{-1} \mathrm{~K}^{-1}$ & $\lambda_{\perp c}^{*} \mathrm{Wm}^{-1} \mathrm{~K}^{-1}$ & $\lambda_{/ / u c}^{*} \mathrm{Wm}^{-1} \mathrm{~K}^{-1}$ & $\lambda_{/ / c}^{*} \mathrm{Wm}^{-1} \mathrm{~K}^{-1}$ & $\frac{D_{l u c}^{*}}{D}$ & $\frac{D_{\perp c}^{*}}{D}$ & $\frac{D_{/ / u c}^{*}}{D}$ & $\frac{D_{/ / c}^{*}}{D}$ & $i \mathrm{~A} / \mathrm{cm}^{2}$ & $R H_{c h} \%$ & $T_{b p}^{\circ} \mathrm{C}$ & $\eta$ \\
\hline 0.25 & 0.25 & 4. & 6.64 & 0.25 & 0.25 & 0.5 & 0.5 & 1.5 & 90 & 80 & 1 \\
\hline
\end{tabular}

direction by at least about a factor 2, e.g. [38], [42], because of a reduced tortuosity effect. Thus, $D_{/ / c}^{*} / D=0.5$. Assuming no significant change in the tortuosity in the through plane direction between the compressed region and the uncompressed one, we take $D_{\perp u c}^{*} \mid D=D_{\perp c}^{*} / D=0.25$. It is assumed that the in-plane coefficient is slightly greater in the uncompressed region with $D_{/ / u c}^{*}=0.7 D$.

With the local conductivity defined as specified in Table 1, the pore network effective diffusion coefficients are of course $D_{\perp c}^{*}$, $D_{/ / c}^{*}, D_{\perp \text { unc }}^{*}, D_{/ / \text {unc }}^{*}$.

The species conservation equation at each network node is expressed as:

$\sum_{j=1}^{n} g_{i j}\left(x_{v, j}-x_{v, i}\right)=0$.

where $n$ is the number of neighbors to considered pore $i ; n=6$ for the cubic network depicted in Fig. 6.

Boundary conditions must be specified in order to solve numerically the system given by Eq. (6). As illustrated in Fig. 4 and also in Fig. 7, the relative humidity $R H$ is imposed in the channel taking as reference saturation vapour partial pressure $p_{v s}$ at $T_{b p}$, i.e. at the temperature of the coldest region in the channel. The vapour partial pressure imposed along the GDL - channel interface is therefore $p_{v}=R H_{c h} p_{v s}\left(T_{b p}\right)$. Thus $x_{v c h}=R H_{c h} p_{v s}\left(T_{b p}\right) / P_{\text {ref }}$ at this interface. A zero flux condition is imposed at the rib - GDL interface while spatially periodic boundary conditions are imposed on the lateral sides of the computational domain. At the GDL inlet (catalyst layer or MPL - GDL interface), the vapour flux is imposed using Eq. (1) assuming a uniform distribution of the current density at the GDL inlet. Hence, the flux given by Eq. (1) is distributed over the GDL inlet proportionally to each inlet throat cross section surface area.

The numerical solution of Eq. (6) with the associated boundary conditions gives $x_{v}$ at each node of network, and therefore the vapour partial pressure at each node of network: $p_{v i, j, k}=x_{v i, j, k} P_{r e f}$. We then compute the local relative humidity at each node of network as $R H_{i, j, k}=p_{v i, j, k} / p_{v s}(T(i, j, k))$.

No condensation occurs when $R H_{i, j, k}<1$ in each node of network, which means that all the water coming from the catalyst layer can be transported in vapour phase across the GDL. Condensation is assumed to occur when $R H_{i, j, k}>\eta$ at least at one node in the network. For most of the simulations presented later in the paper, we have taken $\eta=1$. Thus no supersaturation is assumed. It often happens that the condensation criterion is met not in a single node but at several nodes in the network. Under these circumstances, several options are certainly possible. We decide to proceed step by step considering first only one first condensation

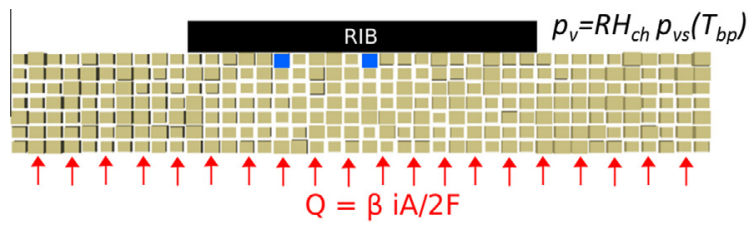

Fig. 7. Sketch of nucleation step. Sketch of two nucleation pores (in blue) under the rib. (For interpretation of the references to colour in this figure legend, the reader is referred to the web version of this article.) node, the one corresponding to $\max \left(R H_{i, j, k}, R H_{i, j, k}>\eta\right)$. At this node, we impose $R H_{i, j, k}=1$, or more exactly the mole fraction $x_{v}$ corresponding to $R H_{i, j, k}=1$. We solve again the problem expressed by Eq. (6) taking into account the new boundary condition $R H_{i, j, k}=1$ at the first condensation node. This gives a new field $R H_{i, j, k}$ and we check whether there are new nodes such that $R H_{i, j, k}>\eta$. If yes this procedure is repeated until all the condensation nodes are identified.

This gives the initial distribution of liquid nodes for the growth step. The nucleation step is schematically illustrated in Fig. 7

\subsection{Liquid cluster growth algorithm}

For simplicity, each liquid node identified at the end of nucleation step is assumed fully saturated by liquid. Another option would be to first compute the condensation - evaporation rate and to determine the filling time of each initial liquid pores. Testing this option is left for a future work. If two of such liquid nodes are first neighbours, they belong to the same cluster. Thus, a first step consists in identifying all the liquid clusters formed at the end of nucleation step, where a cluster is defined as a group of connected liquid pores. Starting from this initial distribution of liquid clusters, the growth step is performed using the following algorithm:

(1) Determine and label the different water clusters. If two pores - totally or partially saturated in liquid water - are adjacent, they belong to the same cluster.

(2) Compute the vapour molar fraction field $x_{v}$ from the solution of the system represented by Eq. (6). In each liquid pore (of coordinates $\left.x_{i}, y_{i}, z_{i}\right)$ in contact with a gas node, the local saturation pressure is imposed. Hence $x_{v}=p_{v s} / P_{\text {ref }}$, where $p_{v s}=p_{v s}\left(T\left(x_{i}, y_{i}, z_{i}\right)\right)$. The boundary conditions on the sides of computational domain are the same as the ones presented in Section 5.1

(3) If the vapour partial pressure computed in step 2 is greater than the local vapour saturation pressure in some gaseous pores, then identify the pore among those pores corresponding to $\max \left(R H_{i, j, k}, R H_{i, j, k}>\eta\right)$ and impose $x_{v}=p_{v s}\left(T\left(x_{i}, y_{i}, z_{i}\right)\right) /$ $P_{\text {ref }}$ in the corresponding pore.

(4) Go back to \#2 and repeat steps \#2 and \#3 until there is no gaseous pore anymore such that $R H_{i, j, k}>\eta$.

(5) Compute the molar condensation rate $F_{k}$ at the boundary of each liquid cluster. This is performed from the computation of the vapour molar fraction field $x_{v}$ within the pores occupied by the gas phase in a manner similar to the computation of cluster evaporation flux in drying simulations, e.g. [43].

(6) Apply the invasion percolation rule, i.e. [44], at each cluster, i.e. determine the throat of larger diameter along the boundary of each liquid cluster.

(7) Compute the invasion time $t_{k}$ of each cluster $k$, i.e. the time required to fully invade the pore adjacent to the throat determined in \#6: $t_{k}=c_{\ell} V_{a d k} / F_{k}$, where $V_{a d k}$ is the volume remaining to invade in the considered pore, $c_{\ell}$ is the mole concentration of liquid water.

(8) Compute the time step $d t=\min \left(t_{k}\right)$.

(9) Fully invade the pore corresponding to $d t$ and update the volume of liquid in the invaded pore in the other clusters. 


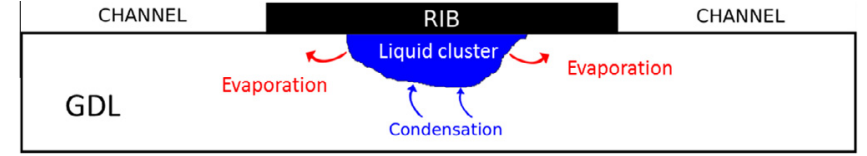

Fig. 8. Illustration that the computed liquid water distribution corresponds to a steady state where the evaporation rate at the boundary of each liquid cluster exactly compensates the condensation rate.

(10) Go back to \#1 and repeat the different steps 1-9 until a steady-state solution is reached.

It should be clear that both evaporation and condensation occur during the growth of a liquid cluster as well as when the steadystate is reached. The steady - state is actually reached when the evaporation rate exactly balances the condensation rate at the boundary of each liquid cluster within the numerical accuracy of our model. This is sketched in Fig. 8.

\section{Condensation diagram}

\subsection{Localization and occurrence of first condensation spots}

According to the algorithm presented in Section 5, condensation occurs when $R H_{i, j, k}>\eta$ somewhere in the network. To get insight into the most likely place of first condensation, one can look at the distribution of $R H$ within the network computed assuming no liquid in the network: $R H(x, y, z)=x_{v}(x, y, z) P_{r e f} / p_{v s}(T(x, y, z))$. For the boundary conditions considered in the present article, an example of computed local relative humidity distribution is depicted in Fig. 9

As can be seen from Fig. 9, the most likely place of incipient condensation is in the region of GDL located below the rib. A noticeable feature is that the local relative humidity is quite high not only right below the rib but all over the thickness of the GDL below the rib consistently with the temperature field depicted in Fig. 5 showing that all the region below the rib is colder.

\subsection{Condensation phase diagram}

Another interesting aspect lies in the range of parameters leading to possible condensation. In our model, there are four operational parameters: the current density $i$, the operating total gas pressure $P_{r e f}$, the operating temperature $T_{b p}$ and the relative humidity in the channel $R H_{c h}$. Solving the vapour diffusion problem in the GDL using the pore network model and varying $R H_{c h}$ and $i$ for given temperature and pressure (we take $P_{\text {ref }}=1.5 \mathrm{bar}$ and $T_{b p}=80^{\circ} \mathrm{C}$ throughout this paper) led to the condensation diagram depicted in Fig. 10. To construct the diagram we impose a current density and progressively increase $R H_{c h}$ starting from a relatively low value, computing the vapour partial pressure field in the network for each considered pair $\left(i, R H_{c h}\right)$. The procedure is stopped when the PN computation of vapour partial pressure field in the network indicated the presence of a first condensation spot $(R H(x, y, z) \geqslant 1$ in one pore within the GDL). This corresponds to the

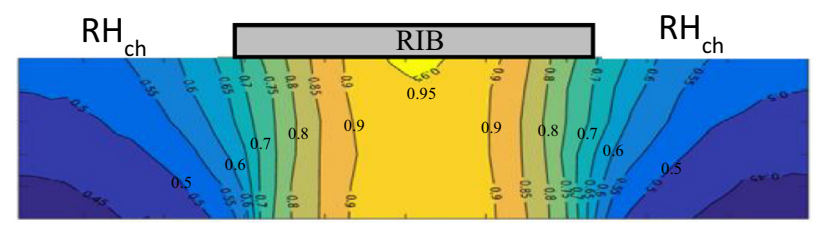

Fig. 9. Example of local relative humidity distribution in a $x-z$ plane cross section of the network in the absence of condensation. This field was computed for $i=1 \mathrm{~A} /$ $\mathrm{cm}^{2}, T_{b p}=80^{\circ} \mathrm{C}$ and $R H_{c h}=50 \%$. Note that the through-plane scale is dilated for clarity. The GDL appears much thicker than it is actually.

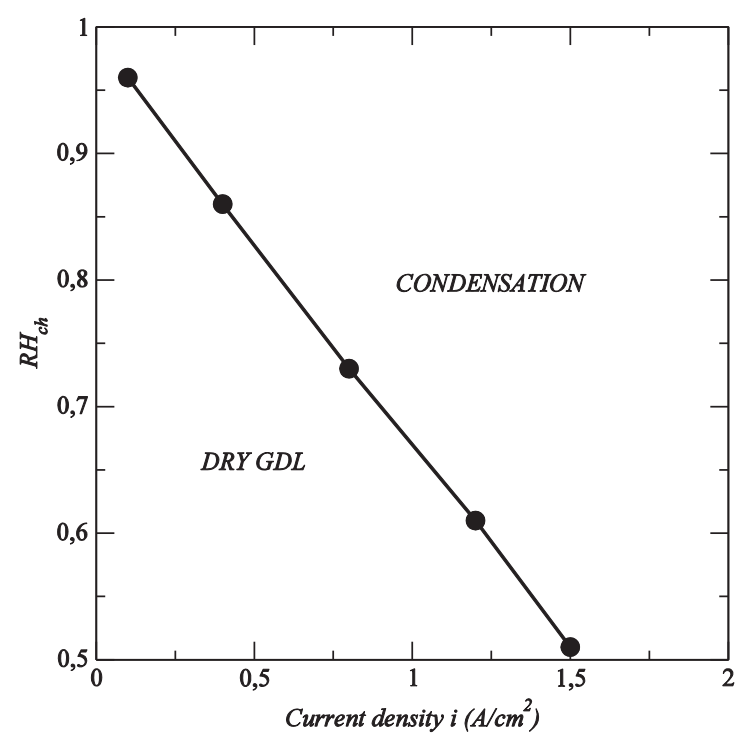

Fig. 10. Condensation diagram obtained for the polarization curve depicted in Fig. 2 and $T_{b p}=80^{\circ} \mathrm{C}$ and $P_{r e f}=1.5$ bar. The water produced in the catalyst layer can be entirely transferred in vapour phase through a fully dry GDL for the operating points located below the curve. Liquid water formation occurs in the GDL for the operating points located above the curve.

determination of one point of the curve shown in Fig. 10. The procedure is then repeated varying $i$ so as to obtain the complete curve.

This diagram specifies the operating conditions for which condensation must occur, i.e. it is not possible to transfer all the water produced in the catalyst layer by diffusion in vapour phase across a dry GDL network when $i$ and $R H_{c h}$ correspond to a point located in the condensation region of the diagram. Conversely, as pointed out for instance in [31], it is possible to transfer all the water produced in vapour phase without liquid formation in the GDL for the points of the diagram located below the dotted black line in Fig. 10.

The condensation diagram depicted in Fig. 10 is in qualitative agreement with the results reported in [37] from in-situ liquid water distribution visualizations obtained using neutron radiography. As shown in [37], no liquid water was detected in the cathode GDL for sufficiently low relative humidity and/or current density. However, the results reported in [37] also indicate that the condensation occurrence depends not only on the channel relative humidity on the cathode side but also on the relative humidity in the channel on the anode side. In the present model, such an effect could be taken into account by modifying the coefficient $\beta$ in Eq. (1) with the relative humidity on the anode side (the greater the relative humidity on the anode side, the greater $\beta$ ). Naturally, a more satisfactory solution would be to develop a modelling fully coupling the anode side and the cathode side.

\section{Liquid water distribution}

In this section, we discuss the phase distribution obtained in the GDL using the condensation PNM. The objective is also to compare the simulations to various experimental results reported in the literature. To facilitate the discussion, Table 1 summarizes the values of GDL parameters specified in the previous sections and defines a reference case $\left(i=1.5 \mathrm{~A} / \mathrm{cm}^{2}, R H_{c h}=90 \%\right)$. The liquid water distribution is discussed from 3D images of distribution, as exemplified in Fig. 11, through plane and transverse liquid water saturation profiles. For a given layer of pores in the in plane directions (i.e. in a $x-y$ plane), the through plane saturation is defined as the fraction of the pore volume in this layer occupied by liquid water. Similarly, for a given layer of pores in a $y-z$ plane (see Fig. 3 ), the 

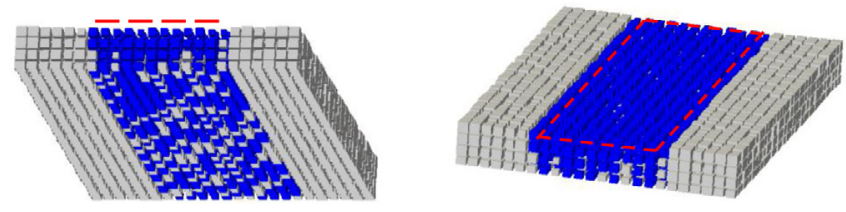

Fig. 11. Two images of the liquid water (in blue) distribution in the GDL for the reference case $\left(i=1.5 \mathrm{~A} / \mathrm{cm}^{2}, R H_{c h}=90 \%\right)$. The red dashed lines materialize the position of the rib. (For interpretation of the references to colour in this figure legend, the reader is referred to the web version of this article.)

transverse saturation is defined as the fraction of the pore volume in this layer occupied by liquid water. The transverse saturation profile provides information on how the liquid water is distributed between the region of the GDL under the rib and the regions under the channels whereas the through plane saturation profile indicates how the liquid is distributed over the GDL thickness.

\subsection{Separation rib-channel}

Fig. 11 shows the phase distribution in the GDL obtained for the reference case. As we shall see, this distribution is representative of other conditions in current density and channel relative humidity.
The striking feature in Fig. 11 is the net separation between the rib region and the channel region. The region of the GDL below the channels is dry whereas the liquid water content in the region below the rib is quite high. Quite interestingly, this strong separation effect is also seen in several previous works using neutron radiography to image in situ the liquid water distribution in a GDL, e.g. $[36,38,45,46]$. This is interpreted as a strong indication in favour of the condensation scenario (assumption \#2) considered in the present article. In other terms, the liquid-vapour phase change phenomena are central here to explain the observed separation.

\subsection{Impact of channel relative humidity}

Figs. 12 and 13 show the impact of the channel relative humidity. As in the experiments, e.g. [35], the greater is the relative humidity, the greater is the amount of liquid water in the GDL.

As can be seen from Fig. 12 and Fig. 13(b), liquid water is concentrated in the region of the GDL located under the rib. The liquid water region simply tends to spread laterally as $R H_{c h}$ is increased. The variation of the through plane saturation profile with $R H_{c h}$ reported in Fig. 13(a) is in qualitative agreement with the results reported in [35].

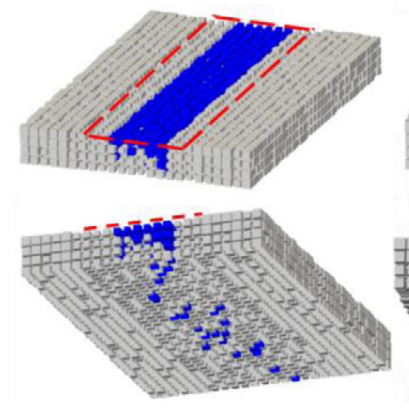

$R H_{c h}=60 \%$
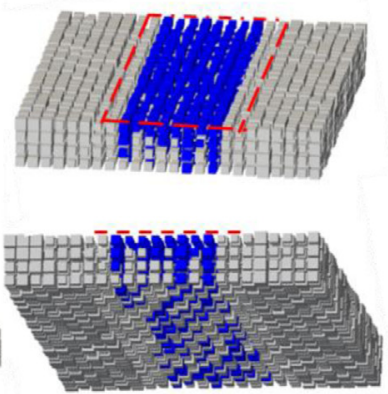

$R H_{c h}=70 \%$

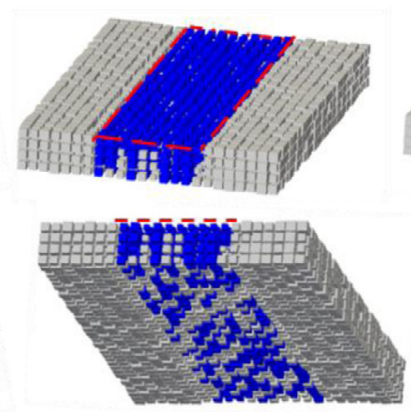

$R H_{c h}=80 \%$
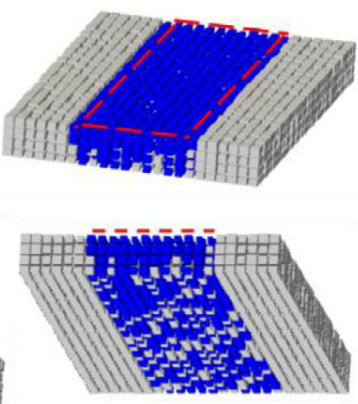

$R H_{c h}=90 \%$

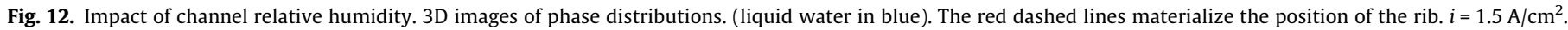
(For interpretation of the references to colour in this figure legend, the reader is referred to the web version of this article.)

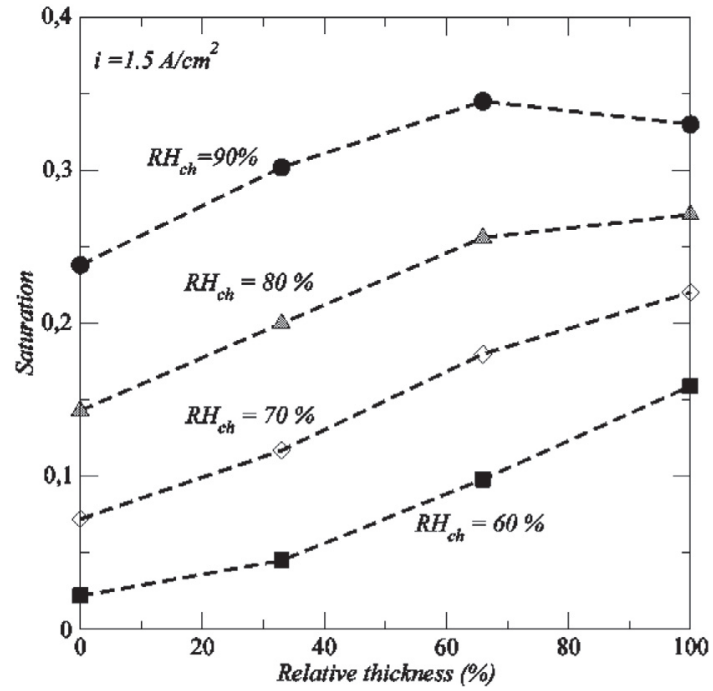

(a) Through plane

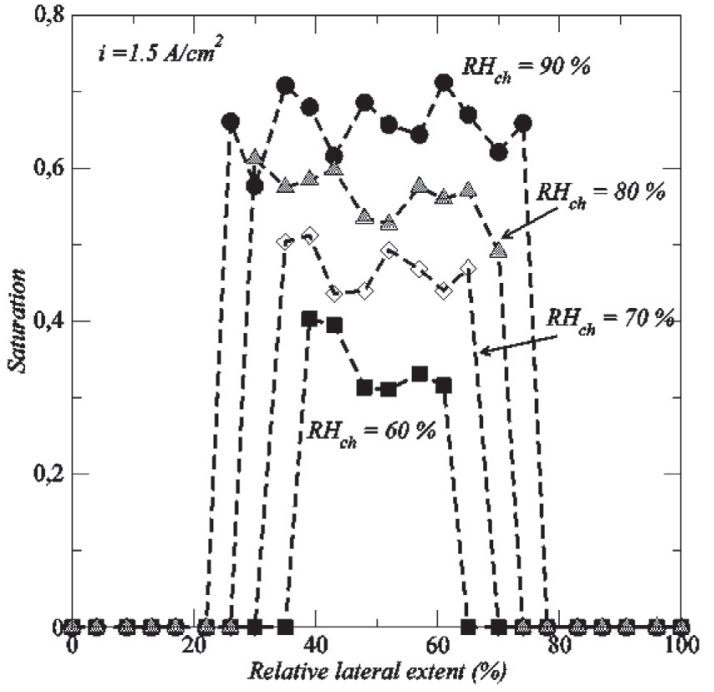

(b) In plane

Fig. 13. Impact of relative humidity: (a) through plane saturation profile, (b) in plane saturation profile. 
As in [35] we can observe a change in the shape of the throughplane saturation profile, from monotonically varying for a sufficiently low relative humidity to concave as the relative humidity is increased. However, the shape of the monotonic profiles is somewhat different, the saturation is greater on the rib-channel side in the PNM simulations whereas the opposite in observed in [35] (see Fig. 7 in this reference).

\subsection{Impact of current density}

The impact of current density is illustrated in Figs. 14 and 15. The change in the through-plane saturation profile (Fig. 15a) is again qualitatively similar to the results reported in [35] (see Fig. 9 in this reference), where however a lower temperature was considered $\left(60^{\circ} \mathrm{C}\right.$ instead of $80^{\circ} \mathrm{C}$ in the PNM simulations). One observes a change from a monotonic profile to a concave one as the current density is increased. Similarly as for the impact of the current density Section 7.2), the region where liquid water is present is the region under the rib and its lateral extent increases with the current density (as illustrated in Figs. 14 and 15(b)).

\subsection{Impact of nucleation parameter}

As shown in Figs. 16 and 17, the nucleation parameter $\eta$ has an interesting impact. First, it affects the shape of both the through plane and in plane saturation profiles. This is illustrated in
Fig. 17. Note in particular in Fig. 17(a) that the saturation on the rib - channel side becomes lower that the saturation at the GDL inlet (i.e. at the GDL - catalyst layer interface) when the nucleation parameter is increased whereas the opposite is observed for $\eta=1$. Interestingly a saturation lower on the rib-channel side than at the GDL inlet is also observed in certain saturation profiles reported in [35].

As depicted in Fig. 17(b), the liquid water remains mostly confined in the region of the GDL below the rib but tends to invade a bit the region under the channel as this parameter is increased.

This is further illustrated in Fig. 16, which interestingly shows that the liquid water occupancy within the region below the rib tends to become sparser as $\eta$ is increased. However, it must be noted that modifying the nucleation parameter does not change the main features of predicted liquid water distribution, i.e. the separation effect and as regards the impact of current density and relative humidity discussed previously.

\section{Discussion}

Compared with previous works on liquid water formation based on PNM, the condensation PNM simulations can be considered as much more consistent with the available experimental data on liquid water distributions in GDLs. Yet the model could be improved. The temperature field computation could be improved by taking
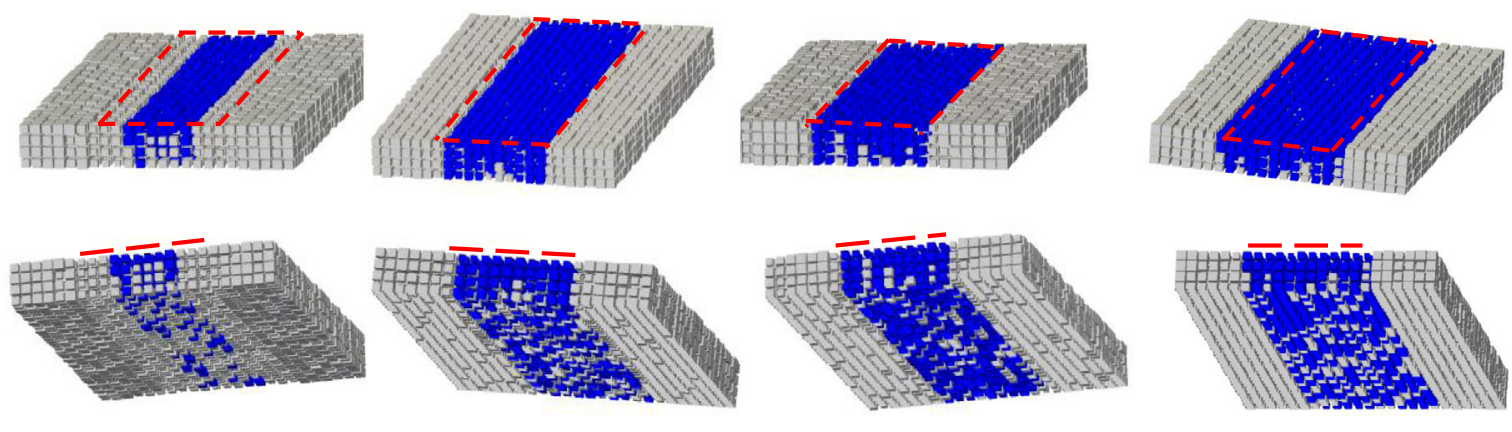

$$
0.4 \mathrm{~A} / \mathrm{cm}^{2}
$$

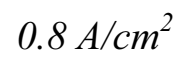

$$
1.2 \mathrm{~A} / \mathrm{cm}^{2}
$$

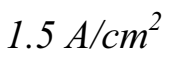

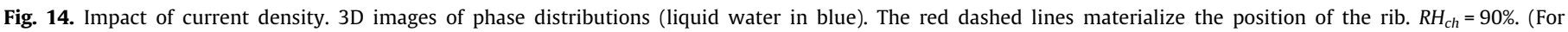
interpretation of the references to colour in this figure legend, the reader is referred to the web version of this article.)

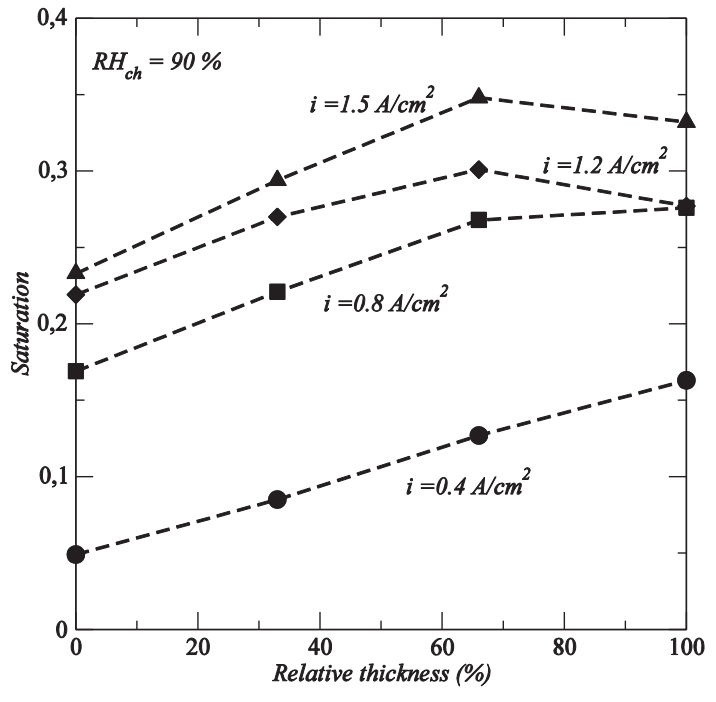

(a)

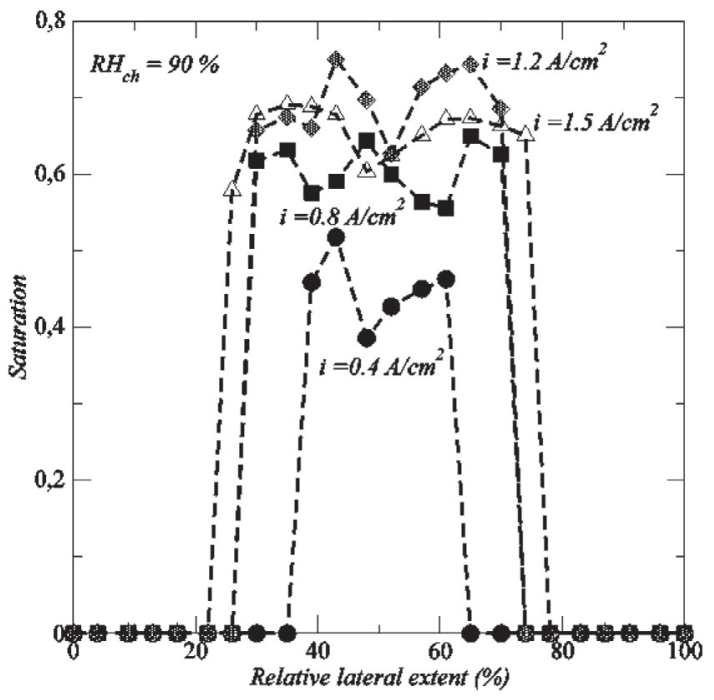

(b)

Fig. 15. Impact of current density: (a) through plane saturation profile, (b) in plane saturation profile. 

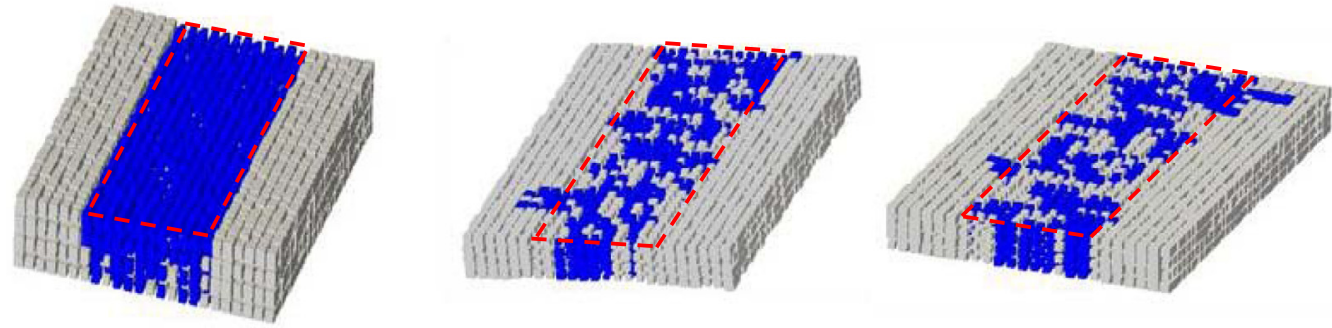

$\eta=1$

$\eta=1.05$

$\eta=1.1$

Fig. 16. Impact of nucleation parameter. Images of the distribution of the liquid water (in blue) in the GDL for the reference case $(\eta=1)$ and for $\eta=1.05$ and $\eta=1.1 ; i=1.5 \mathrm{~A} /$ $\mathrm{cm}^{2}, R H_{c h}=90 \%$. The red dashed lines materialize the position of the rib. (For interpretation of the references to colour in this figure legend, the reader is referred to the web version of this article.)

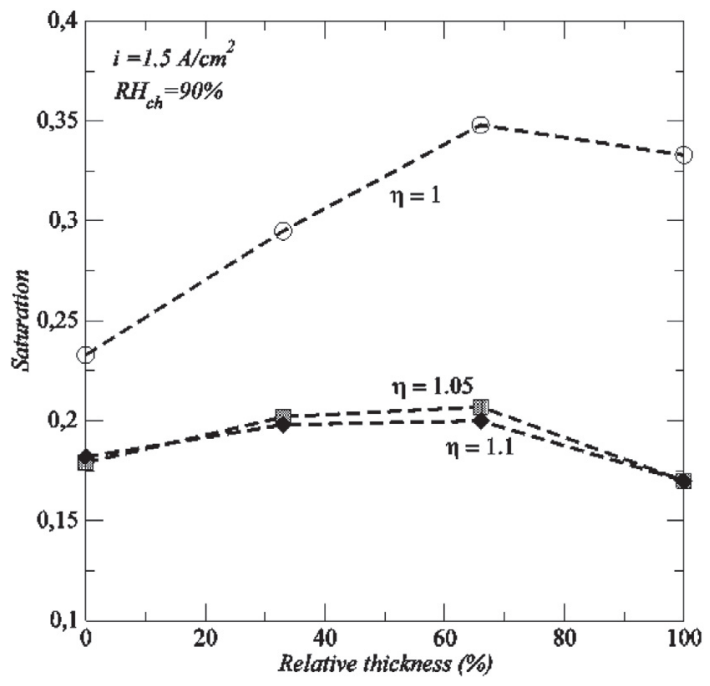

(a)

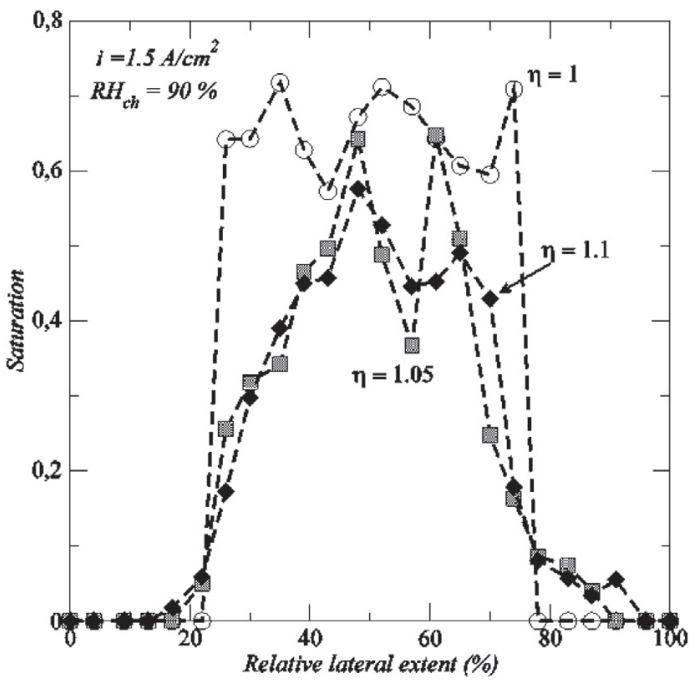

(b)

Fig. 17. Impact of nucleation parameter: (a) through plane saturation profile, (b) in plane saturation profile. $i=1.5 \mathrm{~A} / \mathrm{cm}^{2}, \mathrm{RH} \mathrm{ch}=90 \%$.

into account the impact of liquid water on thermal conductivity and the consideration of the latent heat transfer.

Since the gas mixture on the cathode side is made of air, oxygen and water vapour, the modelling of the diffusion transport could be improved by fully considering the gas phase as a ternary mixture. The oxygen transport does not play any role in the model considered in the present paper. This is so because we consider the current density distribution at the GDL inlet as an input data. Note in passing that the current density was spatially uniform at the GDL inlet in our simulations. However, tests with non-uniform distributions (lower at the entrance of rib region and greater at the entrance of channel regions as well as conversely) did not lead to significantly different results from those reported in the present paper. A more satisfactory solution would be to develop a modelling coupling the transfers in the GDL with the transfers in the catalyst layer (and the MPL). In such a model, the current density distribution would be an output of the model and not an input. Furthermore, this would enable one to predict the impact of liquid water formation on the fuel cell performance, i.e. the polarization curve. Work in this direction in progress. The model presented and discussed in the present paper is nevertheless a key element for such a significantly more involved modelling.

\section{Conclusion}

Condensation in a GDL unit cell was simulated using a condensation pore network model (PNM). The liquid - gas distribution in the GDL resulting from the condensation process was found to be markedly different from the distribution typically reported in most of previous PNM studies on two-phase flow in GDL assuming that the occurrence of water in the GDL results from the capillary controlled invasion in liquid phase from the adjacent MPL or catalyst layer.

The trends obtained with the condensation PNM are in quite good agreement with several experimental observations reported in the literature such as the impact of current density and channel relative humidity on the through plane saturation profiles. Also the simulations are in quite good agreement with the experimental observations indicating a strong separation of the water content under the ribs and under the channels. In our simulations as well liquid water gathers preferentially under the rib.

Our simulations confirm that water produced in the catalyst layer can be transferred through a perfectly dry GDL for a sufficiently low current density and/or a sufficiently low relative humidity in the channel. By contrast, liquid water forms in the GDL as the results of the condensation of the water vapour for a sufficiently high channel relative humidity and/or a sufficiently large current density. As illustrated in this paper, this can be summarized via the concept of condensation diagram.

A major outcome is also that the water transfer is strongly coupled to the heat transfer since the temperature variations within the GDL are a crucial aspect for the computation of the condensation process. The fact that condensation is a major process explaining the formation of water in GDL can now be considered as firmly 
established, at least for an operating temperature of $80^{\circ} \mathrm{C}$. Further works are needed to study the mechanisms of water formation in GDL for lower temperatures.

In summary, we believe that the proposed model opens up new perspectives for understanding the water transfers in protons exchange membrane fuel cells and the associated water management and aging issues as well as for designing more efficient GDLs.

\section{Acknowledgements}

The authors gratefully acknowledge the funding from the EU project IMPALA ("IMprove Pemfc with Advanced water management and gas diffusion Layers for Automotive application", project number: 303446) within the Fuel Cells and Hydrogen Joint Undertaking (FCHJU).

\section{References}

[1] B. Straubhaar, J. Pauchet, M. Prat, Water transport in gas diffusion layers of PEM fuels cells in presence of a temperature gradient. Phase change effect, Int. J. Hydrogen Energy 40 (35) (2015) 11668-11675.

[2] J. Eller, T. Rose, F. Marone, M. Stampanoni, A. Wokaun, F.N. Büchi, Progress in in situ X-ray tomographic microscopy of liquid water in gas diffusion layers of PEFC, J. Electrochem. Soc. 158 (8) (2011) B963-B970.

[3] M. Rebai, M. Prat, Scale effect and two-phase flow in a thin hydrophobic porous layer. application to water transport in gas diffusion layers of PEM fuel cells, J. Power Sources 192 (2008) 534-543.

[4] L. Hao, P. Cheng, Lattice Boltzmann simulations of water transport in gas diffusion layer of a polymer electrolyte membrane fuel cell, J. Power Sources 195 (12) (2010) 3870-3881.

[5] K. Seidenberger, F. Wilhelm, J. Haußmann, H. Markötter, I. Manke, J. Scholta, Grand canonical Monte Carlo study on water agglomerations within a polymer electrolyte membrane fuel cell gas diffusion layer, J. Power Sources 239 (1) (2013) 628-641.

[6] P.K. Sinha, C.Y. Wang, Pore-network modeling of liquid water transport in gas diffusion layer of polymer electrolyte fuel cell, Electrochim. Acta 52 (2007) 7936-7945.

[7] J.T. Gostick, M.A. Ioannidis, M.W. Fowler, M.D. Pritzker, Pore network modelling of fibrous gas diffusion layers for polymer electrolyte membrane fuel cells, J. Power Sources 173 (2007) 277-290.

[8] B. Markicevic, A. Bazylak, N. Djilali, Determination of transport parameters for multiphase flow in porous gas diffusion electrodes using a capillary network model, J. Power Sources 171 (2007) 706-717.

[9] G. Luo, Y. Ji, C.-Y. Wang, P.K. Sinha, Modeling liquid water transport in gas diffusion layers by topologically equivalent pore network, Electrochim. Acta 55 (2010) 5332-5341.

[10] J.T. Gostick, Random pore network modeling of fibrous PEMFC gas diffusion media using Voronoi and Delaunay tessellations, J. Electrochem. Soc. 160 (8) (2013) F731-F743.

[11] A. Bazylak, V. Berejnov, B. Markicevic, D. Sinton, N. Djilali, Numerical and microfluidic pore networks: towards designs for directed water transport in GDLs, Electrochim. Acta 53 (26) (2008) 7630-7637.

[12] J. Hinebaugh, Z. Fishman, A. Bazylak, Unstructured pore network modeling with heterogeneous PEMFC GDL porosity distributions, J. Electrochem. Soc. 157 (2010) B1651-B1657.

[13] K.J. Lee, J.H. Nam, C.J. Kim, Pore-network analysis of two-phase water transport in gas diffusion layers of polymer electrolyte membrane fuel cells, Electrochim. Acta 54 (2009) 1166-1176.

[14] K.J. Lee, J.H. Kang, J.H. Nam, C.J. Kim, Steady liquid water saturation distribution in hydrophobic gas-diffusion layers with engineered pore paths: an invasion-percolation pore-network analysis, J. Power Sources 195 (11) (2010) 3508-3512.

[15] K.J. Lee, J.H. Kang, J.H. Nam, Liquid water distribution in hydrophobic gasdiffusion layers with interconnect rib geometry: an invasion-percolation porenetwork analysis, Int. J. Hydrogen Energy 39 (2014) 6646-6656.

[16] S.P. Kuttanikkad, M. Prat, J. Pauchet, Pore-network simulations of two-phase flow in a thin porous layer of mixed wettability: application to water transport in gas diffusion layers of proton exchange membrane fuel cells, J. Power Sources 196 (2011) 1145-1155.

[17] L. Ceballos, M. Prat, Invasion percolation with multiple inlet injections and the water management problem in proton exchange membrane fuel cells, J. Power Sources 195 (2010) 825-828.

[18] L. Ceballos, M. Prat, P. Duru, Slow invasion of a nonwetting fluid from multiple inlet sources in a thin porous layer, Phys. Rev. E 84 (2011) 056311.

[19] L. Ceballos, M. Prat, Slow invasion of a fluid from multiple inlet sources in a thin porous layer: influence of trapping and wettability, Phys. Rev. E 87 (2013) 043005.
[20] R. Wu, Q. Liao, X. Zhu, H. Wang, Impacts of the mixed wettability on liquid water and reactant gas transport through the gas diffusion layer of proton exchange membrane fuel cells, Int. J. Heat Mass Transfer 55 (2012) 25812589.

[21] R. Wu, X. Zhu, Q. Liao, R. Chen, G.-M. Cui, Liquid and oxygen transport in defective bilayer gas diffusion material of proton exchange membrane fuel cell, Int. J. Hydrogen Energy 38 (10) (2013) 4067-4078.

[22] M. Fazeli, J. Hinebaugh, A. Bazylak, Investigating inlet condition effects on PEMFC GDL liquid water transport through pore network modeling, J. Electrochem. Soc. 162 (7) (2015) F661-F668.

[23] C. Qin, Water transport in the gas diffusion layer of a polymer electrolyte fuel cell: dynamic pore-network modeling, J. Electrochem. Soc. 162 (9) (2015) F1036-F1046.

[24] E.F. Medici, J.S. Allen, Evaporation, two phase flow, and thermal transport in porous media with application to low-temperature fuel cells, Int. J. Heat Mass Transfer 65 (2013) 779-788.

[25] J. Hinebaugh, A. Bazylak, Condensation in PEM fuel cell gas diffusion layers: a pore network modelling approach, J. Electrochem. Soc. 157 (10) (2010) B13821390.

[26] R. Alink, D. Gerteisen, Modeling the liquid water transport in the gas diffusion layer for polymer electrolyte membrane fuel cells using a water path network, Energy 6 (2013) 4508-4530.

[27] J.H. Nam, M. Kaviany, Effective diffusivity and water-saturation distribution in single- and two layer PEMFC diffusion medium, Int. J. Heat and Mass Transfer 46 (2003) 4595-4611.

[28] S. Basu, C.Y. Wang, K.S. Chen, Phase change in a polymer electrolyte fuel cell, J. Electrochem. Soc. 156 (6) (2009) B748-B756.

[29] D.A. Caulk, D.R. Baker, Heat and water transport in hydrophobic diffusion media of PEM fuel cells, J. Electrochem. Soc. 157 (8) (2010) B1237-B1244.

[30] D.A. Caulk, D.R. Baker, Modeling two-phase water transport in hydrophobic diffusion media for PEM fuel cells, J. Electrochem. Soc. 158 (4) (2011) B384B393.

[31] J.P. Owejan, J.E. Owejan, W. Gu, T.A. Trabold, T.W. Tighe, M.F. Mathias, Water transport mechanisms in PEMFC gas diffusion layers, J. Electrochem. Soc. 157 (2010) B1456-B1464.

[32] A. Thomas, G. Maranzana, S. Didierjean, J. Dillet, O. Lottin, Thermal effect on water transport in proton exchange membrane fuel cell, Fuel Cells 12 (2) (2012) 212-224.

[33] A. Thomas, G. Maranzana, S. Didierjean, J. Dillet, O. Lottin, Measurements of electrode temperatures, heat and water fluxes in PEMFCs: conclusions about transfer mechanisms, J. Electrochem. Soc. 160 (2) (2013) F191-F204.

[34] A. Thomas, G. Maranzana, S. Didierjean, J. Dillet, O. Lottin, Thermal and water transfer in PEMFCs: investigating the role of the microporous layer, Int. J. Hydrogen Energy 39 (6) (2014) 2649-2658.

[35] J.M. LaManna, S. Chakraborty, J.J. Gagliardo, M.M. Mench, Isolation of transport mechanisms in PEFCs using high resolution neutron imaging, Int. J. Hydrogen Energy 39 (2014) 3387-3396.

[36] P. Boillat, D. Kramer, B.C. Seyfang, G. Frei, E. Lehmann, G.G. Scherer, A. Wokaun, Y. Ichikawa, Y. Tasaki, K. Shinohara, In situ observation of the water distribution across a PEFC using high resolution neutron radiography, Electrochem. Commun. 10 (2008) 546-550.

[37] P. Oberholzer, P. Boillat, Local characterization of PEFCs by differential cells: systematic variations of current and asymmetric relative humidity, J. Electrochem. Soc. 161 (1) (2014) F139-F152.

[38] N. Zamel, X. Li, Effective transport properties for polymer electrolyte membrane fuel cells - with a focus on the gas diffusion layer, Prog. Energy Combust. Sci. 39 (2013) 111-146.

[39] T. Agaesse, A. Lamibrac, F. Buechi, J. Pauchet, M. Prat, Comparison between pore network simulations and X-Ray tomography images of ex-situ water distributions in a gas diffusion layer of proton exchange membrane fuel cell, J. Power Sources (2015) submitted for publication.

[40] A. Lamibrac, J. Roth, M. Toulec, F. Marone, M. Stampanoni, F.N. Büchi, Characterization of liquid water saturation in gas diffusion layers by X-ray tomographic microscopy, J. Electrochem. Soc. 163 (2016) F202-F209.

[41] L. Mottet, T. Coquard, M. Prat, Three dimensional liquid and vapour distribution in the wick of capillary evaporators, Int. J. Heat Mass Transfer 83 (2015) 636-651.

[42] P.A. Garcia-Salaberri, G. Hwang, M. Vera, A.Z. Weber, J.T. Gostick, Effective diffusivity in partially-saturated carbon-fiber gas diffusion layers: effect of through-plane saturation distribution, Int. J. Heat Mass Transfer 80 (2015) 319-333.

[43] M. Prat, Recent advances in pore-scale models for drying of porous media, Chem. Eng. J. 86 (2002) 153-164.

[44] D. Wilkinson, J.F. Willemsen, Invasion percolation: a new form of percolation theory, J. Phys. A Math. Gen. 16 (1983) 3365-3376.

[45] Y. Tabuchi, T. Shiomi, O. Aoki, N. Kubo, K. Shinohara, Effects of heat and water transport on the performance of polymer electrolyte membrane fuel cell under high current density operation, Electrochim. Acta 56 (1) (2010) 352-360.

[46] A. Turhan, S. Kim, M. Hatzell, M.M. Mench, Impact of channel wall hydrophobicity on through-plane water distribution and flooding behavior in a polymer electrolyte fuel cell, Electrochim. Acta 55 (2010) 2734-2745. 